\title{
Role of Histopathology in Autoimmune Hepatitis
}

\author{
Dina G. Tiniakos ${ }^{a-c}$ John G. Brain ${ }^{a, b} \quad$ Yvonne A. Bury ${ }^{b}$ \\ ${ }^{\mathrm{a}}$ Institute of Cellular Medicine, Faculty of Medical Sciences, Newcastle University, and ${ }^{\mathrm{b}}$ Department of Cellular \\ Pathology, Royal Victoria Infirmary, Newcastle upon Tyne Hospitals Foundation Trust, Newcastle upon Tyne, UK; \\ 'Laboratory of Histology and Embryology, Medical School, National and Kapodistrian University of Athens, Athens, \\ Greece
}

\section{Key Words}

Autoimmune hepatitis · Autoimmune liver disease .

Diagnosis · Histopathology · Liver biopsy

\begin{abstract}
The diagnosis of autoimmune hepatitis (AIH) is based on a combination of biochemical, immunological and histological features and exclusion of other causes of liver disease. Typical histological features include a chronic hepatitis pattern of injury with portal inflammation and interface activity, predominance of plasma cells in the portal infiltrate, emperipolesis, and hepatocellular rosette formation. Centrilobular injury with prominent hepatocellular necrosis and mononuclear inflammation is now recognised in the histological spectrum of AlH and may represent an early stage of the disease. Liver histology plays a major role in clinical diagnostic scoring systems and is important to confirm or support the clinical diagnosis of AlH. This review focuses on the role of histopathology in $\mathrm{AlH}$ and highlights the contribution of histological interpretation to the diagnosis of $\mathrm{AlH}$, differential diagnosis from other entities, recognition of concurrent liver disease, and identification of the so-called overlap or variant syndromes, and addresses the importance of liver biopsy in the management and prognosis of patients with AlH.

(c) 2015 S. Karger AG, Basel
\end{abstract}

(C) 2015 S. Karger AG, Basel

0257-2753/15/0338-0053\$39.50/0

\section{Introduction}

The diagnosis of autoimmune hepatitis (AIH) is clinico-pathological and is based on a combination of biochemical, immunological and histological features and exclusion of other causes of liver disease. Major criteria include increased serum imunoglobulins (Igs), specific serum autoantibodies and typical or compatible histology, while patient response to immunosuppression is characteristic and supports the diagnosis. On histological grounds, AIH is characterised by non-specific features with no pathognomonic lesions. However, in most cases, AIH can be easily distinguished from the 2 other major autoimmune liver diseases, primary biliary cirrhosis (PBC) and primary sclerosing cholangitis (PSC). Difficulties may arise in the differential diagnosis of 'overlap' syndromes and when the presentation of $\mathrm{AIH}$ is atypical.

This review focuses on the changing role of liver biopsy in AIH and on the contribution of liver histology to the clinical diagnostic scoring systems, the differential diagnosis from other entities, and the diagnosis of $\mathrm{AIH}$ in concurrence with other liver diseases, including the socalled overlap or variant syndromes. Finally, the role of histopathology in AIH patient management and prognosis is briefly addressed.

\section{KARGER 125}

E-Mail karger@karger.com www.karger.com/ddi
Dina G. Tiniakos, $\mathrm{MD}, \mathrm{PhD}$

Institute of Cellular Medicine, Faculty of Medical Sciences, Newcastle University William Leech Building, 4th Floor, Room M4-143

Framlington Place, Newcastle upon Tyne NE2 4HH (UK)

E-Mail dina.tiniakos@ newcastle.ac.uk 


\section{Role of Liver Biopsy in AlH}

Liver histology is important to confirm or support the clinical diagnosis of AIH, as it plays a major role in both older and most recent scoring systems. Most importantly, liver biopsy is significant for the differential diagnosis of $\mathrm{AIH}$, as it may not infrequently highlight an alternative aetiology for the underlying liver tissue injury [1]. In addition, it may identify cases with overlapping features within the spectrum of autoimmune liver diseases and highlight possible concurrent disease, especially now that obesity, diabetes and associated fatty liver disease have reached epidemic proportions. Liver histology is currently the best standard for grading necroinflammatory injury and evaluating the extent of fibrosis (staging) and architectural remodelling in AIH. Furthermore, in contrast to viral hepatitis, liver biopsy is still very important for patient management in $\mathrm{AIH}$, as it provides vital information for treatment decisions at diagnosis and for disease remission or progression during treatment. Baseline histology may also prove invaluable for future therapy decisions, since patients may develop other liver diseases while on immunosuppression. Last but not the least, liver histology at presentation may provide prognostic information for subsequent development of progressive fibrosis/cirrhosis and the risk of liver-related death or transplantation, and may yield predictive information regarding response to treatment [1-3].

\section{Contribution of Histology in the Diagnostic Scoring Systems of AlH}

In the 1999 revised criteria of the International AIH Group (IAIHG) [4], histology played a significant diagnostic role, and moderate or severe interface hepatitis was one of the cornerstones for AIH diagnosis alongside clinical and serological parameters. IAIHG updated and simplified the clinico-pathological criteria in 2008 [5], and liver biopsy histology typical or compatible with AIH was one of the 4 necessary elements for the definite diagnosis of $\mathrm{AIH}$, the other 3 being increased serum IgG levels, presence of serum autoantibodies (cut-off $\geq 1: 40$ ) and absence of markers for viral hepatitis (table 1). Treatment response has been used in separate scores required for a diagnosis of definite or probable AIH before and after treatment $[4,6]$.

The 2008 IAIHG simplified scoring system for AIH condenses liver histology criteria into 3 categories: 'typical' when in addition to interface hepatitis, rosetting and emperipolesis are present, 'compatible' when not all 3 fea-
Table 1. Simplified criteria for the diagnosis of AIH (modified from [5])

\begin{tabular}{lll}
\hline Variable & Cut-off & Points \\
\hline ANA or ASMA & $\geq 1: 40$ & 1 \\
ANA or ASMA & $\geq 1: 80$ & \\
or LKM & $\geq 1: 40$ & $2^{*}$ \\
or SLA & Positive & \\
\hline IgG & $>$ UNL & 1 \\
& $>1.10 \times$ UNL & 2 \\
\hline Liver histology & Compatible with & \\
(evidence of hepatitis & AIH & 1 \\
is a necessary condition) & Typical AIH & 2 \\
\hline Absence of viral hepatitis & Yes & 2 \\
& & $\geq 6:$ probable \\
& & AIH \\
& & AIH definite \\
\hline
\end{tabular}

SLA = Soluble liver antigen/liver pancreas; UNL = upper normal limit. * Sum of points achieved for all autoantibodies (maximum 2 points).

Table 2. Typical and compatible histology for the diagnosis of AIH in the 2008 simplified criteria (modified from [5])

\begin{tabular}{ll}
\hline Diagnostic category & Histological features \\
\hline $\begin{array}{l}\text { Typical AIH* } \\
2 \text { points }\end{array}$ & $\begin{array}{l}\text { Interface hepatitis with lymphocytic or } \\
\text { lymphoplasmacytic portal inflammatory } \\
\text { infiltrates extending into the lobule } \\
\text { Hepatocyte rosette formation } \\
\text { Emperipolesis }\end{array}$ \\
\hline $\begin{array}{l}\text { Compatible } \\
\text { with AIH }\end{array}$ & $\begin{array}{l}\text { Chronic hepatitis with lymphocytic } \\
\text { infiltration without all the features } \\
\text { considered typical }\end{array}$ \\
\hline Atypical 0 points & Evidence of another diagnosis
\end{tabular}

* To be considered typical, all 3 histological features have to be present.

tures are present, and 'atypical' when there is evidence of an alternative diagnosis (table 2). Histological evidence of hepatitis is a necessary element for the diagnosis [5]. Retrospective studies from different geographical areas have shown high specificity and sensitivity of the simplified criteria in diagnosing AIH [7]. However, both the 1999 and the 2008 scoring systems have limitations, notably in diagnosing acute severe $\mathrm{AIH} \mathrm{[8]} \mathrm{or} \mathrm{in} \mathrm{the} \mathrm{presence} \mathrm{of} \mathrm{co-}$
Tiniakos/Brain/Bury 

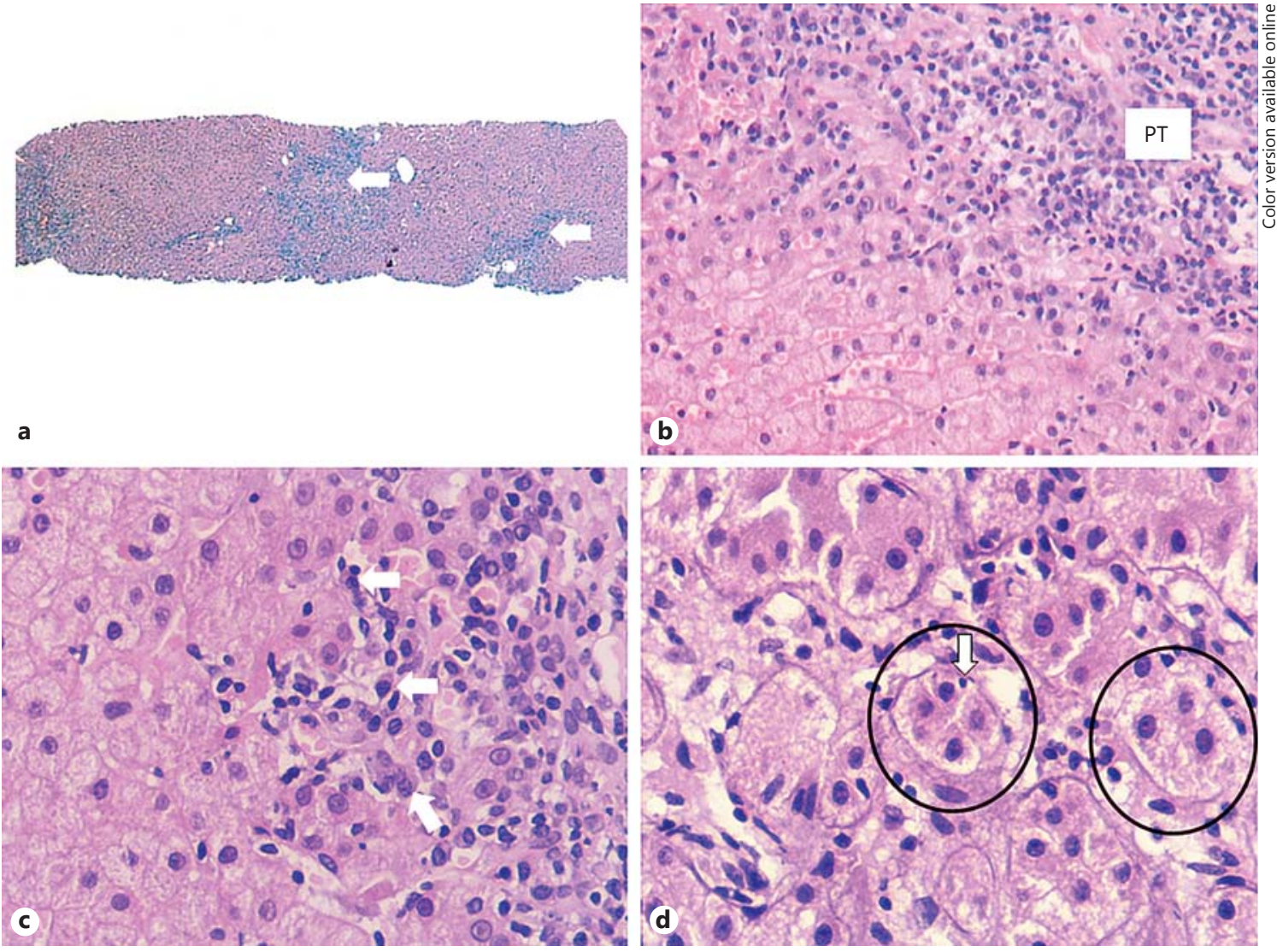

Fig. 1. Typical histological features for the diagnosis of AIH: (a) chronic hepatitis pattern with a mononuclear cell inflammatory infiltrate in portal tracts (arrows), Haematoxylin \& Eosin (H\&E) $\times 25$, (b) interface hepatitis: portal lymphoplasmacytic infiltrate

existing pathological liver processes such as viral hepatitis or nonalcoholic fatty liver disease (NAFLD) [6].

The American Association for the Study of Liver Diseases (AASLD) [6], the British Society of Gastroenterology [9], and the European Association for the Study of the Liver (EASL) [9a] in their most recent guidelines recommend liver biopsy at presentation to establish diagnosis and guide treatment in all patients with suspected AIH. However, using the IAIHG simplified criteria, it is possible to reach a probable diagnosis of AIH even in the absence of liver biopsy or compatible histology. Of course, liver histology compatible with AIH continues to be necessary for a definite diagnosis of AIH. In the absence of positive autoantibodies in repeated tests, liver biopsy is essential to diagnose 'autoantibody-negative' AIH, a variant that presents in up to $20 \%$ of patients and responds equally well to corticosteroid therapy $[6,9]$.

Patients diagnosed as having probable AIH have similar clinical phenotypes and behaviour to those diagnosed extending into the lobule $(\mathrm{PT}=$ portal tract), $\mathrm{H} \& \mathrm{E} \times 200$, (c) higher magnification shows many plasma cells at the portal-parenchymal interface, $\mathrm{H} \& \mathrm{E} \times 400$, (d) hepatocellular rosette formation (black circles) and emperipolesis (arrow), $\mathrm{H} \& \mathrm{E} \times 400$. as definite AIH. Thus, patients with a clinically based diagnosis of probable AIH may not differ from those where AIH was confirmed or supported by liver biopsy [10]. A recent study has questioned the value of liver biopsy in patients with typical laboratory features of AIH, since liver histology did not show any additional or unexpected findings that could affect patient management in the vast majority of cases [11]. However, the significance of liver biopsy in dismissing the diagnosis in patients who otherwise would have been misclassified without a tissue examination was not addressed in this study [12].

\section{Histopathology of AlH}

\subsection{Typical Histological Features of AIH}

The classic histological pattern of AIH is that of chronic hepatitis (fig. 1a). Typical histological features in the 2008 simplified scoring system for AIH include a 
portal mononuclear cell inflammatory infiltrate extending into the lobule (interface hepatitis), emperipolesis and hepatocyte rosetting [5] (table 2; fig. 1). These features are non-specific and may be observed in liver disease of variable aetiology. Emperipolesis and rosette formation appear superior to interface hepatitis and plasma cell-rich infiltrates as histological predictors of $\mathrm{AIH}$ in biopsies of adult patients with chronic hepatitis [13].

The portal inflammatory infiltrate in AIH ranges from mild to severe and includes lymphocytes, histiocytes and, commonly, plasma cells (fig. 1b). A number of eosinophils and neutrophils may also be seen. Predominance of plasma cells in the portal infiltrate (fig. 1c) is typical for AIH but they are present only in $2 / 3$ of biopsies and their absence does not exclude the diagnosis. Usually, a majority of plasma cells in AIH are Ig G-positive by immunohistochemistry, and the IgM/IgG ratio may aid in the differential diagnosis from $\mathrm{PBC}$ in cases with significant bile duct injury (see section 4.2.2) [14]. Interface hepatitis with spill-over of inflammatory cells into the periportal parenchyma (fig. 1c) and hepatocyte necrosis is observed in $84-98 \%$ of the cases, is more prominent in disease flares, and is usually more severe in AIH compared to chronic hepatitis of other aetiology [3].

Emperipolesis, characterised in AIH by the presence of a lymphocyte or rarely a plasma cell within the cytoplasm of hepatocytes (fig. 1d), is reportedly found in 65$78 \%$ of AIH cases $[13,15]$. It occurs more commonly in AIH compared to chronic viral hepatitis, $\mathrm{PBC}$ or druginduced liver injury (DILI) and usually entails CD8+ T lymphocytes. Under physiological circumstances, emperipolesis and subsequent degradation of autoreactive T-lymphocytes is important for the maintenance of immune tolerance. It is thought that interference with this mechanism may result in the accumulation of viable autoreactive T-lymphocytes in the liver and breach of tolerance leading to the development of AIH [16]. In AIH, emperipolesis may induce hepatocyte apoptosis and has been proposed as an additional mechanism of autoimmune-mediated hepatocyte injury. AIH patients with emperipolesis in liver biopsy have significantly higher serum transaminase levels, more severe necroinflammatory activity and more advanced fibrosis [15].

Hepatocellular rosettes, an indication of hepatocellular regeneration following lobular injury, is a diagnostic histological feature of AIH in both the 1999 [4] and 2008 [5] scoring criteria. Rosettes are small groups of hepatocytes arranged around a small, sometimes not visible, central lumen (fig. 1d). The presence and extent of ro- sette formation are better appreciated on reticulin stain. Rosetting is more common in AIH (49\% of cases) compared to chronic viral hepatitis [13]. In paediatric patients, rosette formation has emerged as the most significant histologic predictor of AIH in biopsies with chronic hepatitis [17].

\subsection{Additional Histological Features of AIH}

In AIH, lobular inflammation of variable severity is usually present with scattered necroinflammatory foci (spotty necrosis), confluent and/or bridging necrosis (fig. 2a). Lobular disarray, hepatocyte injury with ballooning and/or apoptosis, and hepatocyte regeneration with 2 -cell thick plates are frequent features. Lymphocytic endophlebitis may be present in a minority of cases $[1,3,13]$. Small collections of macrophages (microgranulomas) and canalicular and/or hepatocellular cholestasis may be found in severe AIH [3]. Similar to hepatitis of other aetiology, fibrosis develops in $\mathrm{AIH}$ as a consequence of healing of tissue injury and may progress from portal/ periportal fibrosis to portal-portal and portal-central fibrous septa formation (bridging fibrosis), and cirrhosis. As fibrosis progresses, proliferating bile ductules at the portal- or septal-parenchymal interface with associated inflamed stroma (ductular reaction) are noted.

\subsubsection{Centrilobular Injury}

Centrilobular injury with prominent hepatocellular necrosis and lymphoplasmacytic inflammation has recently been recognised in the histological spectrum of $\mathrm{AIH}$ (fig. 2b) and is now considered a significant histological feature of the disease. In acute-onset $\mathrm{AIH}$, the presence of centrilobular necrosis supports the diagnosis (see section 4.3.1). Centrilobular necrosis as a predominant finding has been observed in up to $29 \%$ of biopsies with $\mathrm{AIH}$ and it was reported as the only finding in $2-3 \%$ of the cases $[18,19]$. It occurs with similar frequency in patients with and without cirrhosis [19]. Centrilobular necrosis probably represents an early stage of AIH that later may undergo transition to the classical pattern of portal/periportal hepatitis. Rarely, liver injury may remain centrilobular. Centrilobular necrosis is not correlated with a particular clinical phenotype or autoantibody profile and its presence does not alter patient management [20].

\subsubsection{Bile Duct Injury}

It is of note that interlobular bile duct destruction may be observed in $12 \%$ of AIH biopsies [3], while lymphocytic infiltration of bile duct epithelium (lymphocytic
56

Dig Dis 2015;33(suppl 2):53-64 DOI: $10.1159 / 000440747$
Tiniakos/Brain/Bury 

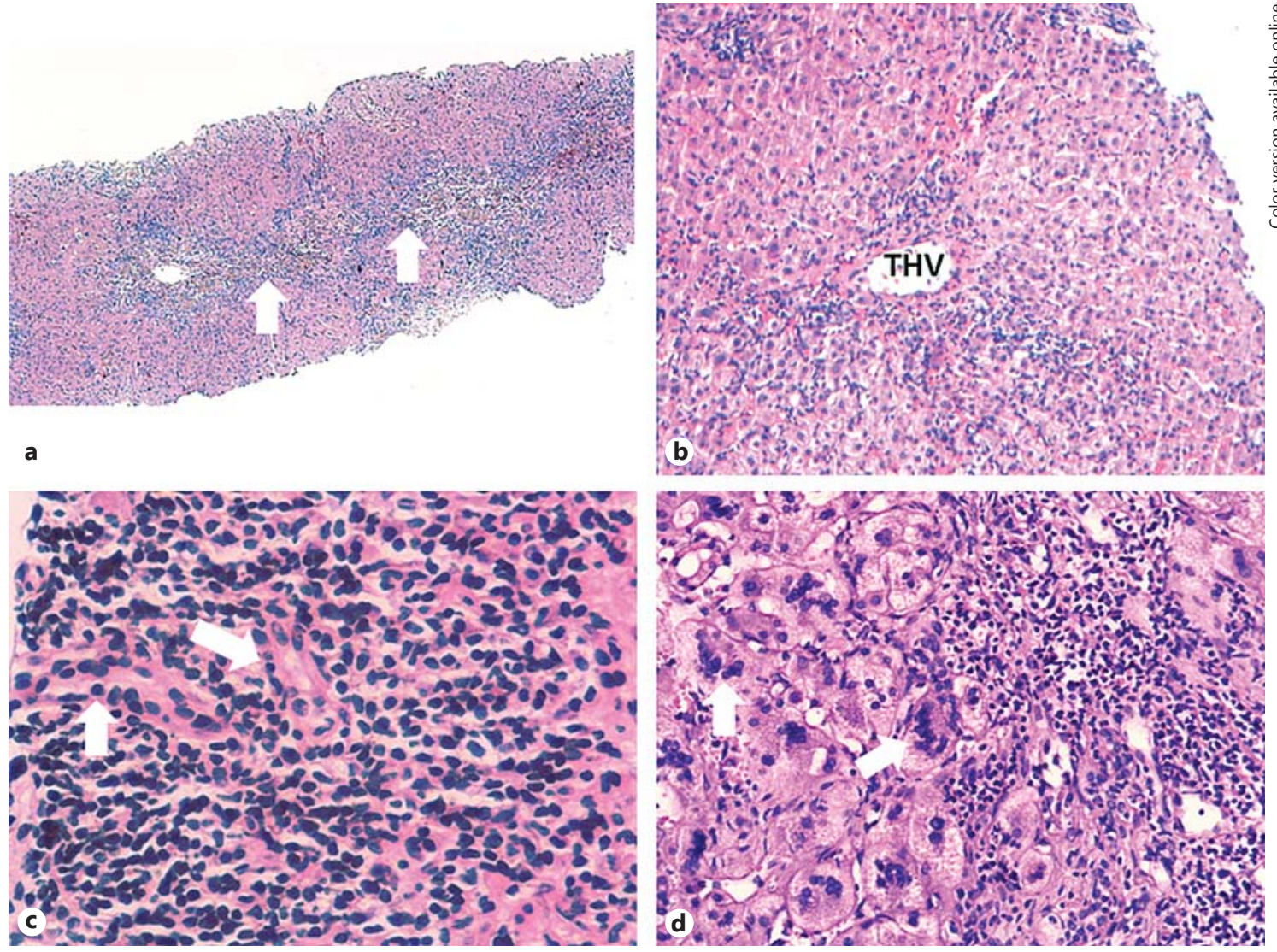

Fig. 2. a Severe AIH with bridging necrosis (arrows), $H \& E \times 40$, (b) centrilobular injury with hepatocellular necrosis and parenchymal lymphoplasmacytic inflammation, THV $=$ Terminal hepatic vein, $\mathrm{H} \& \mathrm{E} \times 100$, (c) dense portal lymphoplasmacytic inflammation and

cholangitis) (fig. 2c) may be present in an additional $12-$ $29 \%$ of the cases $[3,13]$. Therefore, these biliary features are not diagnostic for 'overlap' with $\mathrm{PBC}$ and their presence does not preclude a diagnosis of AIH [13]. Most importantly, the serological profile and response to immunosuppressive therapy of patients with AIH with biliary features are not different compared to those without evidence of biliary injury. In contrast to PBC, bile duct injury in AIH is focal and the IgM/IgG ratio in the portal/ periportal plasmacytic infiltrate, as assessed by immunohistochemistry, is significantly lower than that observed in $\mathrm{PBC}$ where IgM-positive plasma cells predominate [3, 14].

\subsubsection{Granulomas}

Poorly defined granulomas can be observed in 9-11\% of AIH cases [13] but better formed or epithelioid granulomas are rare. Only $4.8 \%$ of granulomatous hepatitis cases are aetiologically related to $\mathrm{AIH}$, while the most com- infiltration of lymphocytes into the bile duct epithelium (lymphocytic cholangitis; arrows), $\mathrm{H} \& \mathrm{E} \times 400$, (d) multinucleated giant hepatocytes (arrows) in the periportal parenchyma in the case of adult AHI, H\&E $\times 200$.

mon aetiology is $\mathrm{PBC}(23.8 \%)$. Granulomas are slightly more common in 'overlap' variants of autoimmune liver disease compared to AIH [21].

\subsubsection{Multinucleated Giant Cells}

Multinucleated giant cells (fig. 2d) may rarely be observed in $\mathrm{AIH}$ as a non-specific idiosyncratic regenerative response to tissue injury. They are the result of either hepatocyte fusion or acytokinetic (amitotic) hepatocyte division. Autoimmune liver disease is the most common cause (40\%) of 'post-infantile giant cell hepatitis' (PIGCH), a rare form of hepatitis, where multinucleated giant hepatocytes predominate. Other less common causes of PIGCH include viruses (mainly paramyxoviruses) and DILI. PIGCH has a variable clinical spectrum ranging from mild acute or chronic icteric hepatitis to rapid progression to cirrhosis and subacute or fatal hepatic failure. Generally, it is a progressive disease with survival rate of $50 \%$ without liver transplantation [22]. 


\subsection{The Many Histological Faces of AIH}

The histological picture of AIH is very diverse and depends on the type of initial presentation, the course of the disease, and the effects of immunosuppressive therapy [1]. In some cases, the typical histological features may be absent; the histological appearances may be modified in disease variants or 'overlap syndromes' while during therapy or in remission, the histology of the disease may be non-specific.

\subsubsection{Acute-Onset and Fulminant AIH}

Acute presentation of $\mathrm{AIH}$ occurs in approximately $20 \%$ of the patients and may have incomplete or atypical phenotype that complicates the recognition of autoimmune aetiology, such as normal serum $\gamma$-globulin and IgG levels or absent conventional autoantibodies $[8,23]$. Czaja et al. [23] has subgrouped acute and fulminant presentation of AIH into chronic AIH with spontaneous exacerbation, chronic AIH with superimposed acute viral or toxic injury, acute-onset 'de novo' AIH, and acute-onset AIH following immune-modulating treatment or liver transplantation.

Acute-onset AIH can resemble an acute viral or toxic hepatitis and liver histology is needed for both diagnosis and treatment planning [24]. Liver biopsy may exclude other aetiology and support the diagnosis of AIH by identifying centrilobular necrosis, with or without interface hepatitis, and other typical features of AIH $[23,25]$. Early liver biopsy is proposed by many investigators for the diagnosis of acute-onset AIH [26]. Histological features of established fibrosis serve as evidence of chronicity in acute-onset AIH.

A minority of patients with acute-onset AIH present with acute liver failure (ALF). In these patients, autoimmune aetiology can be established only in $2-16 \%$ of the cases and in $20 \%$, the cause remains indeterminate [27]. Many of these cases may actually be of autoimmune aetiology but remain undiagnosed because existing diagnostic criteria do not perform well in the acute presentation, and liver biopsy is not performed routinely in ALF due to the risk of haemorrhage. Nevertheless, many authorities believe that information provided by histology may be worth the risk of performing a liver biopsy in indeterminate cases of ALF $[23,28]$. In patients with severe coagulopathy, a transjugular liver biopsy or a mini-laparoscopy with guided liver biopsy may be performed.

Recently, histological criteria for probable autoimmune aetiology have been proposed in ALF patients. These include a distinctive pattern of massive hepatic ne- crosis with centrilobular accentuation, lymphoid follicles, a plasma cell-rich inflammatory infiltrate and central perivenulitis. These features are related to an autoimmune clinical phenotype but are not entirely specific for autoimmune aetiology [25].

\subsubsection{AIH Presenting with Cirrhosis}

Cirrhosis exists at the time of presentation in up to one-third of AIH patients [29-31] and it is often of the macronodular type. AIH-related cirrhosis does not have any pathognomonic histological features $[1,3]$.

An accurate diagnosis of cirrhosis in AIH is important because it influences the choice and dose of immunosuppressive agent(s) and also has prognostic and predictive implications (see section 6) $[9,32]$. Unfortunately, diagnosis of cirrhosis may not be confirmed by liver biopsy in up to $40 \%$ of cases $[1,11]$ due to sampling variability. Small sample size of around 1/50,000 of total liver parenchyma indicates that needle biopsy material may be obtained from a large parenchymal nodule, thus lacking the characteristic fibrous septa and nodular configuration and making histological diagnosis of cirrhosis very difficult. Non-invasive diagnostic modalities for liver fibrosis, such as transient elastography (Fibroscan ${ }^{\circledR}$ ), may falsely show high values of liver stiffness in AIH due to increased inflammatory activity [33].

Hepatocellular carcinoma in AIH has been described only in the context of cirrhosis and has a reported incidence rate of $0.3-1 \%$ per year and a frequency of $1-10 \%$ in cirrhotic AIH patients $[34,35]$.

\subsubsection{Post-Treatment AIH}

Post-treatment AIH has features of chronic hepatitis with fibrosis and shows the absence of either inflammation or mild portal, interface and/or lobular inflammatory activity, depending on treatment response [1]. The aim of the treatment is complete histological remission of hepatic inflammation or minimal residual inflammatory activity (as measured by a histological activity index $\leq 3 / 18)$ [7]. The titres of anti-smooth muscle actin (ASMA) (>1:80) and ASMA antibodies with specificity for F-actin (AAA) $(>1: 40)$ are strongly associated with histological features of disease activity in treated AIH [36]. Histological response lags behind improvement of clinical parameters by 3-8 months and treatment should continue for this period. In $55 \%$ of cases, liver biopsy shows the evidence of interface activity despite normal clinical and laboratory findings. The AASLD [6] and EASL [9a] guidelines recommend biopsy to prove histological remission post-treatment.
58

Dig Dis 2015;33(suppl 2):53-64 DOI: $10.1159 / 000440747$
Tiniakos/Brain/Bury 
Relapse is common (up to $50 \%$ of cases) particularly in patients with childhood onset and/or concurrent associated autoimmune diseases, and can occur early or as late as 20 years after withdrawal of therapy $[6,7,9]$. The histopathology of relapsed AIH is similar to that of untreated AIH [3].

Regression of fibrosis in post-treatment biopsies is reported in up to $57 \%$ of patients [37]. Regression of cirrhosis has been documented in AIH patients associated with reduction of accompanying inflammation, while failure to suppress inflammation worsens fibrosis [38].

\subsection{Serological Variants of AIH}

As with other autoimmune liver diseases, the presence of specific autoantibodies is not related to pathogenesis of AIH. Classic serology for AIH is based on anti-nuclear antibodies (ANA), anti-liver and kidney microsomal-1 (LKM-1) and anti-liver cytosol type-1 antibodies, dividing patients into 2 main groups: (1) type I AIH with ANA and/or ASMA and (2) type II AIH with anti-LKM-1 and/ or anti-liver cytosol type-1 antibodies. Isolated anti-soluble liver antigen/liver pancreas antibodies can be found in AIH patients [7].

\subsection{1 'Autoantibody-Negative' AIH}

'Autoantibody-negative' AIH is defined by the absence of ANA, ASMA, anti-LKM and AMA antibodies at presentation and occurs in $10-20 \%$ of AIH cases. 'Autoantibody-negative' AIH resembles type I AIH in all respects, including histology, aside from serological marker profile. This includes a good response to treatment with steroids [39]. Liver histology is required for the diagnosis of 'autoantibody-negative' AIH. In acute presentation, centrilobular necrosis and a plasma-cell-rich inflammatory infiltrate supports the diagnosis. In chronic disease, the presence of interface hepatitis and absence of findings of other chronic liver disease, such as steatosis, granulomas, biliary injury and siderosis are compatible with $\mathrm{AIH}$ [39].

\subsubsection{Anti Mitochondrial Antibody-Positive AIH}

O'Brien et al. [40] followed up 15 patients who presented with classical biopsy-confirmed AIH but were found to be anti-mitochondrial antibody-M2 (AMA) positive. None of these patients had histological features of PBC, nor did any of them develop PBC on follow-up and the clinical course was that of AIH. It is unclear at this stage whether these patients represent a subtype of $\mathrm{AIH}$ or whether treatment with corticosteroids prevents the subsequent development of PBC or an 'overlap' syn- drome. The management of patients with AMA-positive AIH without biliary injury on biopsy is similar to that of classical AIH patients $[6,9,9 a]$.

\subsection{Histological Grading and Staging Systems}

Grading of inflammatory activity and staging of fibrosis in AIH can be performed using the systems developed for chronic viral hepatitis. Simple 4-tier grading systems like the Batts and Ludwig [41], Scheuer [42] and Metavir [43] are easy to apply in routine practice and have lower inter-observer variability. The more detailed modified histological activity index grading system (range 0-18) [44] that provides separate numeric scores for the severity of lobular inflammation, portal inflammation and interface activity, and the amount and pattern of parenchymal necrosis, is more frequently used for research purposes. The selection of a grading/staging histological system depends on personal preference and agreement between the pathologist and clinician. However, when a specific system is selected, it is important to ensure that it is used consistently and to name the system clearly in the histopathology report.

Staging fibrosis in acute-onset AIH may be difficult, as care should be taken not to overestimate the extent of fibrosis. Areas of bridging necrosis and parenchymal collapse with newly formed collagen that may easily regress with treatment may erroneously appear as fibrotic septa on common collagen stains (Sirius red fast green, van Gieson, Masson trichrome). In these cases, the presence of elastic fibres highlighted by an orcein stain will help in identifying established mature fibrous tissue and accurately stage the disease.

\section{Differential Diagnosis of AlH}

The pattern of injury and typical features of AIH are non-specific; therefore, the histological differential diagnosis is very broad. Liver biopsy is important to exclude other entities, assess concurrent disease, and identify variant/'overlap' syndromes.

\subsection{Exclusion of Other Entities}

In AIH cases with an acute hepatitis pattern of injury, the differential diagnosis mainly includes acute viral hepatitis and DILI. In cases with a chronic hepatitis pattern, chronic viral hepatitis, other autoimmune diseases (see section 5.3), and in young patients, Wilson disease should be considered. The latter is excluded based on laboratory investigations indicating normal copper metabolism. 
Rarely, when no aetiology may be found after detailed clinical, laboratory and histological investigations, the term idiopathic or cryptogenic chronic hepatitis may be used. Some of these cases may truly have an autoimmune nature ('autoantibody-negative') and response to immunosuppressive therapy may aid diagnosis [3].

\subsubsection{DILI}

Idiosyncratic DILI can mimic the clinical, biochemical and serological phenotype of AIH and may serve as a trigger for induction of persistent AIH. Among patients with classical features of type I AIH, 9\% have DILI-related AIH. Currently, minocycline and nitrofurantoin account for $90 \%$ cases of DILI-related AIH. Other probable causes of DILI-related AIH include diclofenac, $\alpha$-methyl DOPA, hydralazine, and more recently, statins and anti-TNF- $\alpha$ agents such as infliximab [45].

Distinguishing DILI from AIH by histology can be extremely difficult, since there is no individual histologic feature that is pathognomonic of either DILI or AIH. However, a recent international study involving 4 expert hepatopathologists has shown that although there is a significant overlap between the 2 diseases, the pattern of injury may suggest the correct diagnosis, even in the absence of clinical information [46]. A combination of distinct findings, such as the types of inflammatory cells in different areas, severity of injury/inflammation, and presence of cholestasis may help in differentiating DILI vs. AIH. Severe portal plasma cell-rich inflammation (grade $\geq 2$ ), prominent intralobular eosinophils, rosette formation, absence of cholestasis and presence of fibrosis (stage $\geq 1$ ) were all features in favour of AIH. There was a $65 \%$ concordance between histologic and clinical diagnosis, indicating that pathologists can use the pattern of liver injury to suggest the correct diagnosis in most cases of DILI vs. $\mathrm{AIH}$ without knowing the clinical information [46].

\subsubsection{NAFLD}

In non-acute AIH, NAFLD is an important clinical differential diagnosis. Positive autoantibody serology, usually low titres of ANA, ASMA and/or AMA, has been reported in $20-48 \%$ of NAFLD patients [47]. In addition, some elderly female patients with nonalcoholic steatohepatitis (NASH)-related cirrhosis may show increased $\gamma$-globulins. Therefore, using the simplified scoring system for AIH [5], rare patients with NASH may be misdiagnosed as having probable AIH. The presence of autoantibodies in NASH may be an epiphenomenon. However, the possibility of a common pathogenetic link between autoimmunity and chronic inflammation of
$\mathrm{NASH}$, possibly related to advanced age and female gender, has been recently proposed [47].

Liver biopsy has been suggested as a superior diagnostic method in some patients showing the typical serological findings of AIH [48] and can reliably exclude AIH based on the pattern of injury, which is predominantly central-based, absence of significant $(\geq 2)$ portal inflammation with interface hepatitis, and presence of characteristic histological features of NASH discussed in more detail in section 5.2.2.

\subsubsection{Viral Hepatitis}

It is mandatory to rule out infection with hepatotropic viruses before the diagnosis of definite or probable AIH and therefore, serological testing is recommended in all patients with this differential diagnosis. In particular, hepatitis virus $A$ and hepatitis virus $E$ (HEV) infection have been reported as close histological mimickers of $\mathrm{AIH}$, and testing for HEV RNA is also recommended for $\mathrm{AIH}$ patients not responding to immunosuppressive therapy [49].

\subsection{Concurrence of AIH with Other Chronic Liver \\ Disease}

Concurrence of AIH with viral hepatitis creates a very difficult clinical problem, since chronic viral hepatitis is used to exclude AIH in diagnostic scoring systems. This problem is not rare in countries where the prevalence of hepatitis virus $\mathrm{B}(\mathrm{HBV})$ or hepatitis virus $\mathrm{C}(\mathrm{HCV})$ infection is high. Similarly, the increasing global prevalence of NAFLD and alcohol-related toxicity makes concurrence of steatohepatitis $(\mathrm{SH})$ with $\mathrm{AIH}$ a challenging diagnosis. It is very important to recognize chronic liver disease patients with concurrent $\mathrm{AIH}$ because untreated severe $\mathrm{AIH}$ has a very poor prognosis. In these cases, liver biopsy is essential in highlighting the coexistence of $\mathrm{AIH}[6,7,9]$.

\subsubsection{Concurrence with Viral Hepatitis}

Chronic infection with $\mathrm{HBV}, \mathrm{HCV}$ and hepatitis virus $\mathrm{D}$ is associated with diverse immunopathological manifestations, including increased serum IgG, development of autoantibodies (ASMA up to 66\%, ANA up to $41 \%$ in chronic hepatitis $\mathrm{C}$, lower prevalence in chronic hepatitis $\mathrm{B}$ and hepatitis virus $\mathrm{D}$ co-infection), and immune complex-mediated disorders. The pathogenetic link between hepatotropic viruses and autoimmune disease is not as yet clarified, but it is known that chronic viral hepatitis patients who develop overt autoimmune disease are genetically predisposed and/or have been treated with cytokines that can favour autoimmunity like $\alpha$-interferon
Tiniakos/Brain/Bury 
[50]. Serum anti-HEV antibodies are more frequent in $\mathrm{AIH}$ patients than in patients with chronic $\mathrm{HBV} / \mathrm{HCV}$, rheumatoid arthritis or healthy individuals [49].

Chronic viral hepatitis infections concomitant with AIH are often extremely difficult to recognise and the criteria for their diagnosis must be very strict $[6,9,50]$. In these patients, a definite diagnosis of AIH relies on a combination of serological and histological findings, scoring systems, response to therapy and patient outcome [51]. HBV patients with concurrent AIH seem to carry the most favourable prognosis compared to those with $\mathrm{HCV}$ infection probably because of the use of nucleotide analogues, which contrary to a-interferon can control HBV replication without exacerbation of autoimmune phenomena [52].

\subsubsection{Concurrence with Steatohepatitis}

The diagnosis of AIH with concurrent $\mathrm{SH}$ of alcoholic or non-alcoholic aetiology can be challenging. $\mathrm{SH}$ is histologically characterised, in addition to steatosis, by lobular inflammation and hepatocyte ballooning that are nonspecific features usually present in AIH [53]. However, Mallory-Denk body formation and centrilobular 'chicken-wire' sinusoidal fibrosis, features that characterise $\mathrm{SH}$, are not seen in $\mathrm{AIH}$, where fibrosis is portal-based and Mallory-Denk body is not a feature. In addition, the type of hepatocyte ballooning with rarefaction and clarification of the cytoplasm associated with pericellular fibrosis that is common in $\mathrm{SH}$ is not seen in AIH. Therefore, these findings may help the pathologist diagnose concurrent $\mathrm{SH}$ in a patient with otherwise typical histological and serological features of AIH [53]. On the other hand, when assessing biopsies from patients with suspected NAFLD and positive autoantibody serology, the presence of prominent portal-based inflammation that exceeds lobular changes should trigger consideration of AIH.

\subsubsection{Concurrence with Other Metabolic Diseases}

Exclusion of other metabolic diseases, such as alpha1antitrypsin deficiency, haemochromatosis and Wilson disease in patients with chronic hepatitis and a differential diagnosis of $\mathrm{AIH}$, is important and is based on both serological and well-established histological criteria. However, several case reports have described AIH with superimposed or coexisting Wilson disease [54] and up to $17.2 \%$ of patients diagnosed with AIH may have a heterozygous C282Y mutation [55]. Presence of hemosiderin granules and evaluation of their cellular and subcellular distribution patterns are very helpful in assessing concurrent siderosis in $\mathrm{AIH}$ patients.

Role of Histopathology in AIH

\subsection{AIH in Variant/ 'Overlap' Syndromes}

Liver biopsy can identify patients with overlapping features between disorders within the spectrum of autoimmune liver diseases and is particularly useful in patients with biochemical cholestasis. A recent position statement by the IAIHG indicates that these patients should be classified and treated according to the predominant features of their disease into AIH, PBC, PSC, or cholestatic variant/'overlap' syndrome [56]. Therefore, liver biopsy should be performed in all AIH patients with clinical suspicion of overlapping features with an autoimmune cholangiopathy, such as positive AMA, cholestatic biochemical profile or concurrent inflammatory bowel disease, in order to assess bile duct injury or loss. Biliary tree imaging will also aid the diagnosis identifying focal biliary strictures and dilations based on cholangiography (present in $2-11 \%$ of cases) [20]. Seven-13\% of adult AIH patients may show features of AIH-PBC, while 8-17 and $49 \%$ of adult and paediatric autoimmune disease patients, respectively, share features of AIH-PSC [56]. PBC-AIH can also rarely develop sequentially with typical histological and pure clinical features of each disease, but predictive prognostic factors for this condition have not yet been identified [57]. Careful histological examination can support the clinical diagnosis of an 'overlap' or variant syndrome, highlight the predominant disease and guide treatment.

Liver biopsy in AIH-PBC variant/'overlap' cases shows features of both $\mathrm{PBC}$, that is, florid bile duct lesions with granulomatous portal and/or lobular inflammation, ductopenia (loss of interlobular bile ducts in $>50 \%$ of complete portal tracts) and evidence of chronic cholestasis, and also shows features of AIH, that is, significant interface and lobular inflammatory activity. Differential diagnosis from pure AIH that may show focal non-destructive bile duct injury has been discussed in section 4.2.2. In addition, in early stage disease, histological features of chronic cholestasis, with copper-associated protein deposition and keratin 7 immunoreactivity in periportal hepatocytes will support a diagnosis of AIH-PBC variant/'overlap' [3, 58].

In AIH-PSC variant/'overlap' syndromes, liver biopsy should show significant lobular necroinflammatory activity combined with typical features of PSC including concentric periductal 'onion-skin'-like fibrosis, obliterative fibrous cholangitis and ductopenia or cholangiographic evidence of bile duct lesions compatible with PSC. Non-specific changes such as portal oedema, bile duct atrophy, degenerative biliary epithelial changes and portal and interface hepatitis are also seen in PSC. Unfortunately, PSC is a focal disease and the random 
distribution of the typical lesions makes their identification on liver biopsy difficult due to sampling variability $[1,3]$.

\section{The Role of Histopathology in AlH Patient Management and Prognosis}

A liver biopsy at first presentation of AIH facilitates treatment decisions especially in cases with atypical features such as acute-onset $\mathrm{AIH}$, concurrent disease, and variant/'overlap' syndromes [2]. In AIH, histological findings are used as indications for treatment in addition to clinical and laboratory parameters. According to current guidelines, bridging or multilobular necrosis is an absolute indication, while relative indication is interface hepatitis. Treatment is not offered in patients with inactive cirrhosis or mild portal inflammation, with no symptoms and with minimal biochemical abnormalities $[6,7,9]$.

Liver biopsy may give significant prognostic information on cirrhosis development and liver-related death and predictive information on treatment response. At presentation, confluent of bridging necrosis is an indicator of progressive fibrosis or cirrhosis development as well as liver-related death or transplantation. Patients with established cirrhosis tend to have a higher frequency of treatment-related adverse effects and a lower rate of response to treatment [59]. On treatment, persistent inflammation can predict high risk of progressive fibrosis. In a biopsy that is done before treatment withdrawal, any inflammation or prominent portal plasma cells are poor prognostic indicators for relapse $[6,9,60]$.

Data for the prognostic role of cirrhosis at presentation for liver-related death are contradictory. A majority of studies indicate that AIH patients with cirrhosis at presentation $[30,32]$ or those who subsequently develop cirrhosis have a worse outcome [32]. Poorer outcome in cirrhotic patients seems to be determined by the poor treatment response in these patients; yet reversal of fibrosis is also documented in AIH-cirrhotic patients, indicating that progressive and regressive phenotypes may exist. Contrastingly, Ngu et al. [29] found in a retrospective study in New Zealand that cirrhosis at presentation was not associated with a poor prognosis and had no influence on response to initial immunosuppressive treatment. Limiting factors in interpretation of such discrepancies could be different treatment regimens or polygenic differences present in differing geographical locations. More plausibly, these issues arise because of the inherent limitations of retrospective studies.

\section{Epilogue}

Liver biopsy interpretation is essential for the definite diagnosis of AIH and should be performed in all patients with an unexplained liver disease. AIH has different histological presentations with typical features, such as interface hepatitis, plasma cell predominance in the portal inflammatory infiltrate, regenerative rosettes and emperipolesis, or compatible features when only interface hepatitis is observed. Predominant or exclusive centrilobular injury is now recognised in the histological spectrum of AIH and may represent an early stage of the disease.

Careful histological examination and an adequate liver tissue sample will always yield helpful information for diagnosis, differential diagnosis, prognosis and treatment decisions in AIH. Detailed clinical history accompanying the biopsy request form and clinico-pathological correlation are necessary for making the correct histological diagnosis in autoimmune liver disease. Therefore, communication and partnership of clinicians with pathologists are essential for optimal patient management.

\section{Acknowledgements}

DGT is central pathologist for UK-AIH funded by National Institute for Health Research (NIHR) Rare Diseases Translational Research Collaboration and NIHR Newcastle Biomedical Research Centre.

\section{Disclosure Statement}

All authors have nothing to disclose.

References

1 Guindi M: Histology of autoimmune hepatitis and its variants. Clin Liver Dis 2010;14: 577-590.

2 Schiano TD, Fiel MI: To b(iopsy) or not to b(iopsy). Clin Gastroenterol Hepatol 2011;9: 3-4.

3 Washington MK, Manns MP: Autoimmune hepatitis; in Burt AD, Portmann B, Ferrel L (eds): MacSween's Pathology of the Liver. Edinburgh, Churchill Livingstone, 2012, pp 467-490.

4 Alvarez F, Berg PA, Bianchi FB, Bianchi L, Burroughs AK, Cancado EL, et al: International autoimmune hepatitis group report: review of criteria for diagnosis of autoimmune hepatitis. J Hepatol 1999;31:929-938.
62

Dig Dis 2015;33(suppl 2):53-64 DOI: $10.1159 / 000440747$
Tiniakos/Brain/Bury 
5 Hennes EM, Zeniya M, Czaja AJ, Parés A, Dalekos GN, Krawitt EL, Bittencourt PL, Porta G, Boberg KM, Hofer H, Bianchi FB, Shibata M, Schramm C, Eisenmann de Torres B, Galle PR, McFarlane I, Dienes HP, Lohse AW; International Autoimmune Hepatitis Group: Simplified criteria for the diagnosis of autoimmune hepatitis. Hepatology 2008;48:169-176.

6 Manns MP, Czaja AJ, Gorham JD, Krawitt EL, Mieli-Vergani G, Vergani D, Vierling JM; American Association for the Study of Liver Diseases: Diagnosis and management of autoimmune hepatitis. Hepatology 2010:51;21932213.

7 Manns MP, Lohse AW, Vergani D: Autoimmune hepatitis - update 2015. J Hepatol 2015; 62(1 suppl):S100-S111.

8 Weiler-Normann C, Lohse AW: Acute autoimmune hepatitis: many open questions. J Hepatol 2014;61:727-729.

9 Gleeson D, Heneghan MA; British Society of Gastroenterology: British society of gastroenterology (BSG) guidelines for management of autoimmune hepatitis. Gut 2011;60:16111629.

9a EASL: EASL Clinical Practice Guidelines: Autoimmune hepatitis. J Hepatol 2015;63:9711004.

10 Czaja AJ: Comparability of probable and definite autoimmune hepatitis by international diagnostic scoring criteria. Gastroenterology 2011;140:1472-1480.

11 Björnsson E, Talwalkar J, Treeprasertsuk S, Neuhauser M, Lindor K: Patients with typical laboratory features of autoimmune hepatitis rarely need a liver biopsy for diagnosis. Clin Gastroenterol Hepatol 2011;9:57-63.

12 Czaja AJ: Review article: the management of autoimmune hepatitis beyond consensus guidelines. Aliment Pharmacol Ther 2013;38: 343-364.

13 de Boer YS, van Nieuwkerk CM, Witte BI, Mulder CJ, Bouma G, Bloemena E: Assessment of the histopathological key features in autoimmune hepatitis. Histopathology 2015; 66:351-362.

14 Abe K, Takahashi A, Nozawa Y, Imaizumi H, Hayashi M, Okai K, Kanno Y, Watanabe H, Ohira H: The utility of IgG, IgM, and CD138 immunohistochemistry in the evaluation of autoimmune liver diseases. Med Mol Morphol 2014;47:162-168.

15 Miao Q, Bian Z, Tang R, Zhang H, Wang Q, Huang S, Xiao X, Shen L, Qiu D, Krawitt EL, Gershwin ME, Ma X: Emperipolesis mediated by CD8 T cells is a characteristic histopathologic feature of autoimmune hepatitis. Clin Rev Allergy Immunol 2015;48:226-235.

16 Benseler V, Warren A, Vo M, Holz LE, Tay SS, Le Couteur DG, Breen E, Allison AC, van Rooijen N, McGuffog C, Schlitt HJ, Bowen DG, McCaughan GW, Bertolino P: Hepatocyte entry leads to degradation of autoreactive CD8 T cells. Proc Natl Acad Sci U S A 2011; 108:16735-16740.

17 Kumari N, Kathuria R, Srivastav A, Krishnani N, Poddar U, Yachha SK: Significance of histopathological features in differentiating autoimmune liver disease from nonautoimmune chronic liver disease in children. Eur J Gastroenterol Hepatol 2013;25:333-337.

18 Hofer H, Oesterreicher C, Wrba F, Ferenci P, Penner E: Centrilobular necrosis in autoimmune hepatitis: a histological feature associated with acute clinical presentation. J Clin Pathol 2006;59:246-249.

19 Miyake Y, Iwasaki Y, Terada R, Onishi T, Okamoto R, Takaguchi K, Ikeda H, Makino Y, Kobashi H, Sakaguchi K, Shiratori Y: Clinical features of Japanese type 1 autoimmune hepatitis patients with zone III necrosis. Hepatol Res 2007;37:801-805.

20 Czaja AJ: Challenges in the diagnosis and management of autoimmune hepatitis. Can J Gastroenterol 2013;27:531-539.

21 Gaya DR, Thorburn D, Oien KA, Morris AJ, Stanley AJ: Hepatic granulomas: a 10 year single centre experience. J Clin Pathol 2003;56: 850-853.

22 Bihari C, Rastogi A, Sarin SK: Postinfantile giant cell hepatitis: an etiological and prognostic perspective. Hepat Res Treat 2013;2013: 601290.

23 Czaja AJ: Acute and acute severe (fulminant) autoimmune hepatitis. Dig Dis Sci 2013;58: 897-914.

24 Fujiwara K, Fukuda Y, Yokosuka O: Precise histological evaluation of liver biopsy specimen is indispensable for diagnosis and treatment of acute-onset autoimmune hepatitis. J Gastroenterol 2008;43:951-958.

25 Stravitz RT, Lefkowitch JH, Fontana RJ, Gershwin ME, Leung PS, Sterling RK, Manns MP, Norman GL, Lee WM; Acute Liver Failure Study Group: Autoimmune acute liver failure: proposed clinical and histological criteria. Hepatology 2011;53:517-526.

26 Yasui S, Fujiwara K, Yonemitsu Y, Oda S, Nakano M, Yokosuka O: Clinicopathological features of severe and fulminant forms of autoimmune hepatitis. J Gastroenterol 2011;46: 378-390.

27 Bernal W, Lee WM, Wendon J, Larsen FS, Williams R: Acute liver failure: a curable disease by 2024? J Hepatol 2015;62(1 suppl): S112-S120.

28 Lee WM: Etiologies of acute liver failure. Semin Liver Dis 2008;28:142-152.

29 Ngu JH, Gearry RB, Frampton CM, Stedman CA: Predictors of poor outcome in patients with autoimmune hepatitis: a populationbased study. Hepatology 2013;57:2399-2406.

30 Grønbæk L, Vilstrup H, Jepsen P: Autoimmune hepatitis in Denmark: incidence, prevalence, prognosis, and causes of death. A nationwide registry-based cohort study. J Hepatol 2014;60:612-617.

31 Czaja AJ: Cryptogenic chronic hepatitis and its changing guise in adults. Dig Dis Sci 2011; 56:3421-3438.

32 Werner M, Wallerstedt S, Lindgren S, Almer S, Björnsson E, Bergquist A, Prytz H, Sandberg-Gertzén $H$, Hultcrantz R, Sangfelt $P$, Weiland O, Ohlsson B, Danielsson A: Char- acteristics and long-term outcome of patients with autoimmune hepatitis related to the initial treatment response. Scand J Gastroenterol 2010;45:457-467.

33 Romanque P, Stickel F, Dufour JF: Disproportionally high results of transient elastography in patients with autoimmune hepatitis. Liver Int 2008;28:177-178.

34 Czaja AJ: Hepatocellular carcinoma and other malignancies in autoimmune hepatitis. Dig Dis Sci 2013;58:1459-1476.

35 Danielsson Borssén Å, Almer S, Prytz H, Wallerstedt S, Friis-Liby IL, Bergquist A, et al: Hepatocellular and extrahepatic cancer in patients with autoimmune hepatitis - a longterm follow-up study in 634 Swedish patients. Scand J Gastroenterol 2015;50:217223.

36 Couto CA, Bittencourt PL, Porta G, AbrantesLemos CP, Carrilho FJ, Guardia BD, Cançado EL: Antismooth muscle and antiactin antibodies are indirect markers of histological and biochemical activity of autoimmune hepatitis. Hepatology 2014;59:592-600.

37 Serpaggi J, Carnot F, Nalpas P, Canioni D, Guéchot J, Lebray P, Vallet-Pichard A, Fontaine H, Bedossa P, Pol S: Direct and indirect evidence for the reversibility of cirrhosis. Hum Pathol 2006;37:1519-1526.

38 Czaja AJ: Review article: the prevention and reversal of hepatic fibrosis in autoimmune hepatitis. Aliment Pharmacol Ther 2014;39: 385-406.

39 Czaja AJ: Autoantibody-negative autoimmune hepatitis. Dig Dis Sci 2012;57:610-624.

40 O'Brien C, Joshi S, Feld JJ, Guindi M, Dienes HP, Heathcote EJ: Long-term follow-up of antimitochondrial antibody-positive autoimmune hepatitis. Hepatology 2008;48:550556.

41 Batts KP, Ludwig J: Chronic hepatitis. An update on terminology and reporting. Am J Surg Pathol 1995;19:1409-1417.

42 Scheuer PJ: Classification of chronic viral hepatitis: a need for reassessment. J Hepatol 1991;13:372-374.

43 Intraobserver and interobserver variations in liver biopsy interpretation in patients with chronic hepatitis C. The French METAVIR cooperative study group. Hepatology 1994; 20:15-20.

44 Ishak K, Baptista A, Bianchi L, Callea F, De Groote J, Gudat F, Denk H, Desmet V, Korb G, MacSween RN, et al: Histological grading and staging of chronic hepatitis. J Hepatol 1995;22:696-699.

45 deLemos AS, Foureau DM, Jacobs C, Ahrens W, Russo MW, Bonkovsky HL: Drug-induced liver injury with autoimmune features. Semin Liver Dis 2014;34:194-204.

46 Suzuki A, Brunt EM, Kleiner DE, Miquel R, Smyrk TC, Andrade RJ, Lucena MI, Castiella A, Lindor K, Björnsson E: The use of liver biopsy evaluation in discrimination of idiopathic autoimmune hepatitis versus drug-induced liver injury. Hepatology 2011;54:931939. 
47 Tsuneyama K, Baba H, Kikuchi K, Nishida T, Nomoto K, Hayashi S, Miwa S, Nakajima T, Nakanishi Y, Masuda S, Terada M, Imura J, Selmi C: Autoimmune features in metabolic liver disease: a single-center experience and review of the literature. Clin Rev Allergy Immunol 2013;45:143-148.

48 Efe C, Ozaslan E, Purnak T, Ozseker B, Kav T, Bayraktar Y: Liver biopsy is a superior diagnostic method in some patients showing the typical laboratory features of autoimmune hepatitis. Clin Res Hepatol Gastroenterol 2012;36:185-188.

49 Pischke S, Gisa A, Suneetha PV, Wiegand SB, Taubert R, Schlue J, Wursthorn K, Bantel H, Raupach R, Bremer B, Zacher BJ, Schmidt RE, Manns MP, Rifai K, Witte T, Wedemeyer H: Increased HEV seroprevalence in patients with autoimmune hepatitis. PLoS One 2014; 9:e85330.

50 Vergani D, Mieli-Vergani G: Autoimmune manifestations in viral hepatitis. Semin Immunopathol 2013;35:73-85.

51 Efe C, Wahlin S, Ozaslan E, Purnak T, Muratori L, Quarneti C, et al: Diagnostic difficul- ties, therapeutic strategies, and performance of scoring systems in patients with autoimmune hepatitis and concurrent hepatitis $\mathrm{B} / \mathrm{C}$. Scand J Gastroenterol 2013;48:504-508.

52 Rigopoulou EI, Zachou K, Gatselis N, Koukoulis GK, Dalekos GN: Autoimmune hepatitis in patients with chronic $\mathrm{HBV}$ and $\mathrm{HCV}$ infections: patterns of clinical characteristics, disease progression and outcome. Ann Hepatol 2014;13:127-135.

53 Burt AD, Lackner C, Tiniakos D: Diagnosis and assessment of NAFLD: definitions and histopathological classification. Semin Liv Dis 2015, in press.

54 Yener S, Akarsu M, Karacanci C, Sengul B, Topalak O, Biberoglu K, Akpinar H: Wilson's disease with coexisting autoimmune hepatitis. J Gastroenterol Hepatol 2004; 19:114-116.

55 Höhler T, Leininger S, Köhler HH, Schirmacher P, Galle PR: Heterozygosity for the hemochromatosis gene in liver diseases - prevalence and effects on liver histology. Liver 2000;20:482-486.

56 Boberg KM, Chapman RW, Hirschfield GM, Lohse AW, Manns MP, Schrumpf E; Interna- tional Autoimmune Hepatitis Group: Overlap syndromes: the international autoimmune hepatitis group (IAIHG) position statement on a controversial issue. J Hepatol 2011; 54:374-385.

57 Efe C, Ozaslan E, Heurgué-Berlot A, Kav T, Masi C, Purnak T, et al: Sequential presentation of primary biliary cirrhosis and autoimmune hepatitis. Eur J Gastroenterol Hepatol 2014;26:532-537.

58 Kobayashi M, Kakuda Y, Harada K, Sato Y, Sasaki M, Ikeda $\mathrm{H}$, et al: Clinicopathological study of primary biliary cirrhosis with interface hepatitis compared to autoimmune hepatitis. World J Gastroenterol 2014;20:3597-3608.

59 Czaja AJ: Advances in the current treatment of autoimmune hepatitis. Dig Dis Sci 2012;57: 1996-2010.

60 van Gerven NM, Verwer BJ, Witte BI, van Hoek B, Coenraad MJ, van Erpecum KJ, et al; Dutch Autoimmune Hepatitis Working Group: Relapse is almost universal after withdrawal of immunosuppressive medication in patients with autoimmune hepatitis in remission. J Hepatol 2013;58:141-147. 